\title{
Retroperitoneal Suppurative Fistula Caused by Remnant Suture Material in a Poodle Dog
}

\section{Keunyung Kim ${ }^{1}$ \\ Chaeyeong Lee \\ Minyeon $\mathrm{Kim}^{1}$ \\ Hyeonjong Choi ${ }^{1}$ \\ Jeongho Hong ${ }^{1}$ \\ Hyoyeon Kim ${ }^{1}$ \\ Hyojin Park ${ }^{1}$ \\ Na-hyun Kim² \\ Dongbin Lee ${ }^{1}$ \\ Jae-Hoon Lee ${ }^{1, *}$}

${ }^{1}$ Institute of Animal Medicine, College of Veterinary Medicine, Gyeongsang National University, Jinju 52828, Korea

${ }^{2}$ Gyeongnam Department of Environment \& Toxicology, Korea Institute of Toxicology, Jinju 52834, Korea

*Correspondence: jh1000@gnu.ac.kr

\section{ORCID}

Keunyung Kim:

https://orcid.org/0000-0003-0585-4702

Chaeyeong Lee:

https://orcid.org/0000-0003-1927-6763

Minyeon Kim:

https://orcid.org/0000-0003-2547-4803

Hyeonjong Choi:

https://orcid.org/0000-0002-8083-2266

Jeongho Hong:

https://orcid.org/0000-0001-6506-7781

Hyoyeon Kim:

https://orcid.org/0000-0002-4620-6704

Hyojin Park:

https://orcid.org/0000-0002-9942-2136

Na-hyun Kim:

https://orcid.org/0000-0002-9518-1478

Dongbin Lee:

https://orcid.org/0000-0002-2645-4508

Jae-Hoon Lee:

https://orcid.org/0000-0002-8340-3694

Copyright $\odot$ The Korean Society of Veterinary Clinics
Abstract An eight-year-old, $3.4 \mathrm{~kg}$, spayed female poodle dog was referred to Gyeongsang National University Animal Medical Center with obstinate bilateral retroperitoneal subcutaneous abscess. The medical history revealed ovariohysterectomy (OHE), lipoma, and mammary gland tumor excision performed two years ago. The dog presented with left retroperitoneal subcutaneous abscess discharge five months back and was treated by abscess drainage and antibiotics in a local veterinary hospital, but a relapse was reported on discontinuing antibiotics. On physical examination, the dog was febrile $\left(40^{\circ} \mathrm{C}\right)$ and both retroperitoneal masses showed typical inflammatory reaction and purulent discharge. On serum chemistry analysis, hyperproteinemia, hyperglobulinemia, and elevated levels of C-reactive protein were observed. Cytologic examination showed degenerative neutrophils and phagocytic macrophages. Radiological examination revealed encapsulated hypoechoic subcutaneous masses in bilateral abdominal flank and the left flank mass was connected with the caudal pole of left kidney through fistula. Based on the diagnostic examination, exploratory celiotomy was recommended and left nephrectomy, and resection of bilateral masses were performed. During procedure, fistula between caudal pole of left kidney and left subcutaneous abscess was detected and suture material was identified. Resected tissue was histologically examined and diagnosed as suppurative abscess caused by the suture material. This case report describes chronic suppurative foreign body reaction including caudal pole of left kidney and bilateral retroperitoneal subcutaneous masses induced by the suture material used in OHE two years ago and the necessity of computed tomography examination to identify character of mass and extent of surgical resection.

Key words suture material, foreign body, fistula, kidney, dog. 


\section{Introduction}

Inflammatory reaction is elevated by the presence of foreign bodies such as sutures, lint, wood, hair, vegetable matter, and surgical swabs $(3,18,22)$. Small peritoneal foreign bodies get eliminated by circulation and lymphatic absorption. A potent foreign body which cannot be eliminated gets separated with a resultant granuloma or localized abscess (18) or can cause fistula in the skin or other organs $(15,22)$. Unrecognized retroperitoneal abscesses may develop causing continuance of clinical signs, progression of retroperitoneal abscess, urinary obstruction, peritonitis, sepsis, and death (22).

The terms fistula and draining tract are used to explain abnormal passages between internal organs or cavities, or leading from an organ or cavity to the skin $(4,19,20)$. The differential diagnosis of fistula in dogs includes bacterial, fungal, and parasitic infections, as well as neoplastic changes, foreign bodies, and inflammatory and systemic conditions $(11,24)$. A previous report showed that 15 of the 25 cases diagnosed as fistula were because of foreign bodies (19). Another report showed chronic fistula caused by foreign body in five of the six dogs (3).

Retroperitoneal infections and abscesses have clinical significance in dogs. However, these disorders have not been reported in cats. Dogs typically present with uncertain waxing and waning clinical signs, making the diagnosis difficult. Frequent veterinary visits and various treatments may be required for an appropriate diagnosis and treatment plan (22).

In this case report, we present a dog with a relapsing abscess lesion for five months, diagnosed as granulomatous inflammation induced by suture material foreign body reaction and treated with left nephrectomy and mass resection.

\section{Case Report}

An eight-year-old, $3.4 \mathrm{~kg}$, spayed female poodle dog was referred to Gyeongsang National University Animal Medical Center for relapsing bilateral retroperitoneal subcutaneous abscess. The dog had undergone ovariohysterectomy with excision of lipoma, and mammary gland tumor two years prior to admission and left retroperitoneal subcutaneous abscess discharge was detected five months prior to admission. The dog underwent drainage of abscess and antibiotics were administered several times at a local hospital, but the mass relapsed following discontinuation of antibiotics.

On physical examination, in left flank, $2 \times 3 \times 2 \mathrm{~cm}$ mass was palpated. In addition, $3 \times 3 \times 2 \mathrm{~cm}$ mass was palpated in right flank. The masses indicated typical inflammatory re- actions like heat, pain, and swelling. The dog showed normal vital signs except being febrile $\left(40.0^{\circ} \mathrm{C}\right)$.

Normal findings were observed on complete blood count and serum chemistry except for mild hyperproteinemia (8.3 $\mathrm{g} / \mathrm{dL}$, reference range: 5.2-8.2 $\mathrm{g} / \mathrm{dL}$ ), hyperglobulinemia (5.1 $\mathrm{g} / \mathrm{dL}$, reference range: $2.5-4.5 \mathrm{~g} / \mathrm{dL}$ ), and elevated levels of $C$ relative protein (6.4 mg/L, reference range: $0.1-1 \mathrm{mg} / \mathrm{L})$. Cytological examination from both the masses showed infiltrated degenerative neutrophils mainly, plasma cells and macrophages in phagocytosis (Fig. 1).

Radiographic examination showed irregular margin of left kidney. Abdominal ultrasonography revealed hypoechoic change in the bilateral subcutaneous masses. In caudal pole of left kidney protrusion hypoechoic heterogenous change and hyperechoic change in fat tissue with fistula in connection with left subcutaneous mass were found (Fig. 2). Contrast computed tomographic examination was performed to evaluate detail of masses, fistula, and extent of surgical resection and indicated fluid attenuating cavitary lesion in both subcutaneous masses and caudal pole of left kidney (Fig. 3). On examination, diagnosis was made as bilateral retroperitoneal suppurative mass and left mass connected with caudal pole of left kidney through fistula. Exploratory celiotomy to identify cause of the lesion and resection of both masses and left nephrectomy were recommended for treatment of relapsing purulent discharge.

The patient was premedicated with atropine $(0.04 \mathrm{mg} / \mathrm{kg}$, SC; Atropine ${ }^{\circledR}$; Jeil Pharmaceutical Co., Daegu, Korea), butorphanol $(0.2 \mathrm{mg} / \mathrm{kg}$, SC; Butophan injection; Myungmoon Pharm Co., Seoul, Korea), diazepam (0.2 mg/kg, IV; Samjin Diazepam Injection; SamJin Parm Co., Seoul, Korea), and

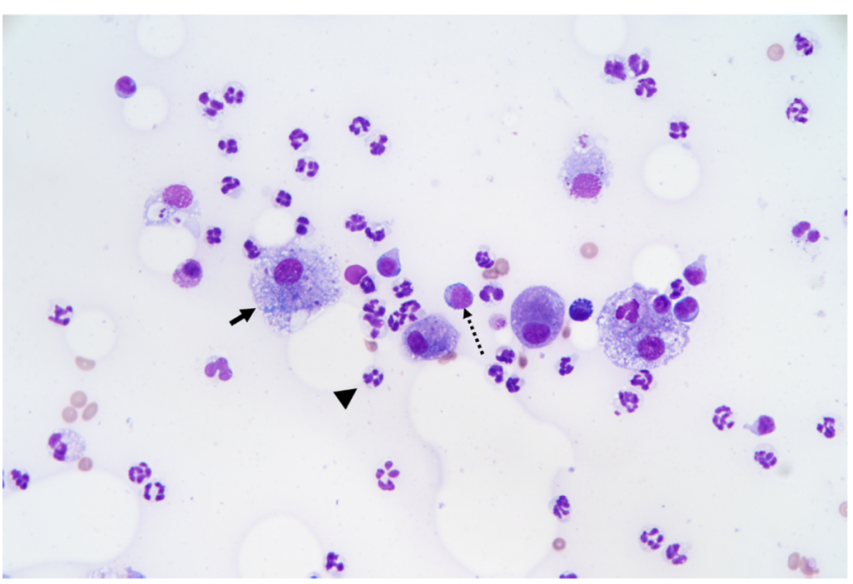

Fig. 1. Cytology of the impression smear in microscopic image. Sample was stained with hematoxylin and eosin (H\&E). Degenerative neutrophil was observed (arrowhead). Macrophages in phagocytosis (arrow) and plasma cells (dotted arrow) were observed (400×). 

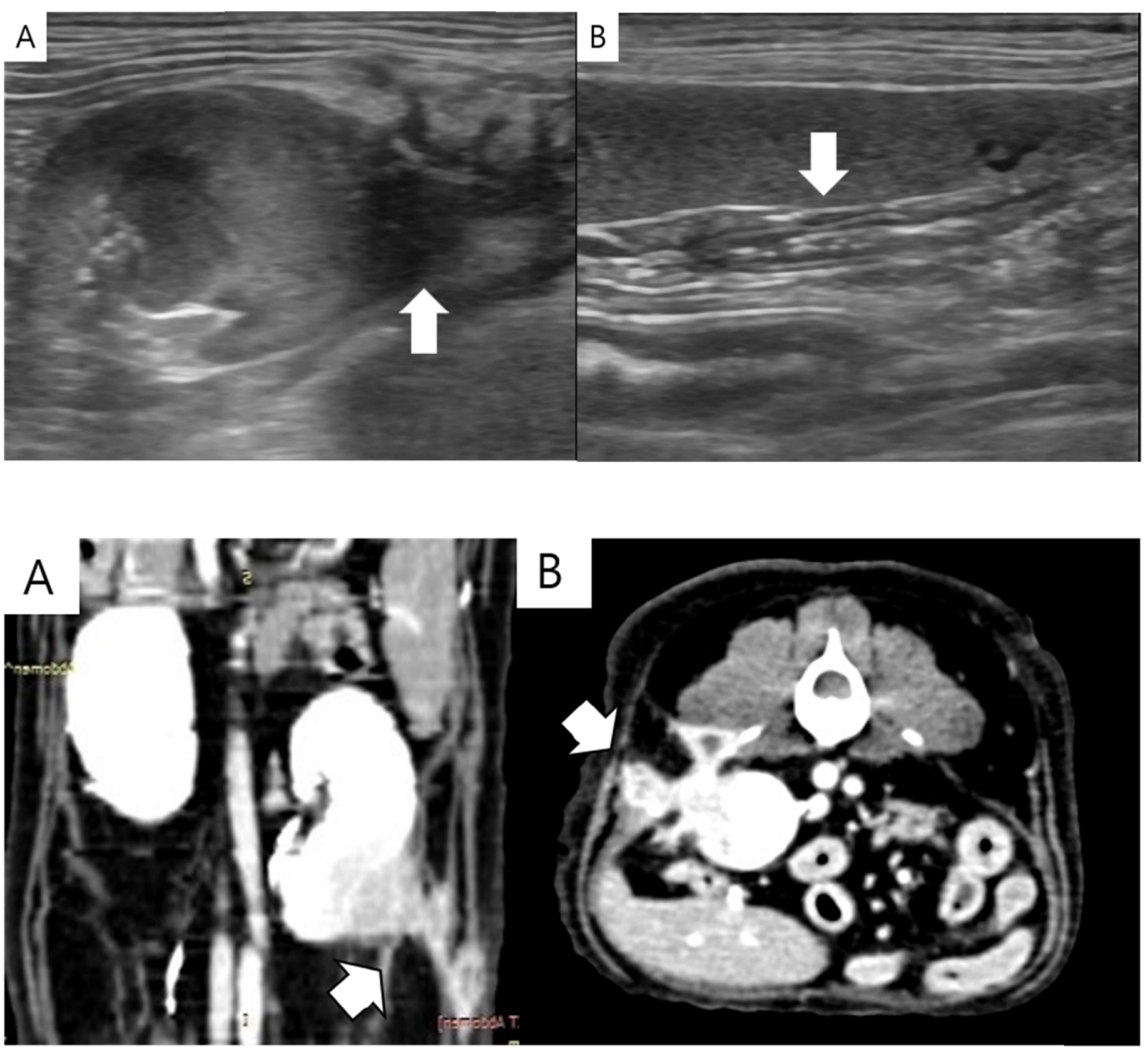

Fig. 2. Ultrasonographic findings in the dog. (A) Irregular shaped hypoechoic heterogenous region (arrow) was observed and the region in protrusion affecting caudal pole of left kidney. (B) The region was connected with left subcutaneous mass through fistula. Hyperechoic change surrounding fistula was observed (arrow).

Fig. 3. Contrast computed tomographic findings in the dog. (A) Fluid attenuating cavitary lesion observed from caudal pole of left kidney through fistula connected left subcutaneous lesion in coronal view (arrow). (B) In sagittal view.
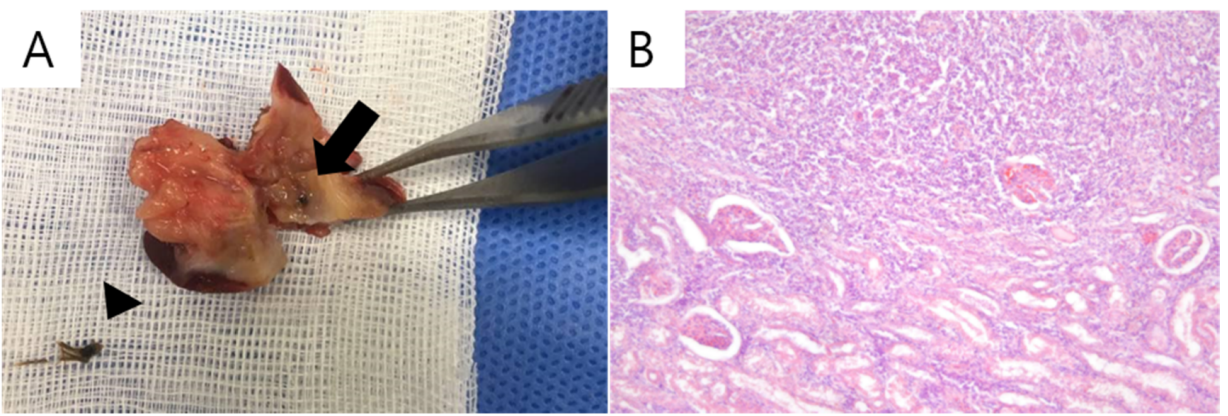

Fig. 4. Gross and histopathological examination of excised left kidney. (A) Remnant suture material in kidney (arrow) and extracted suture material (arrowhead). (B) Granulomatous inflammation affected parenchymal area of the kidney. The sample was stained with H\&E staining observed $(40 \times)$.

cefazolin (25 mg/kg, IV; Cefazol; Hankook Korus Pharm Co., Seoul, Korea). Anesthesia was induced with propofol $(6 \mathrm{mg} /$ kg, IV; Provive; Myungmoon Pharm Co., Seoul, Korea) and maintained with isoflurane (Terrell TM; Piramal Critical Care, USA) inhalation in $100 \%$ oxygen flow rate of $2 \mathrm{~L} / \mathrm{min}$.

The surgical area was aseptically prepared. Both subcutaneous masses were resected marginally. Abdominal incision was extended from xyphoid to caudal up to umbilicus. Surgical site was ensured using Balfour self-retaining retractor. Mild adhesion was identified around caudal pole of left kidney. Renal artery, vein, and involved vessels were sutured with absorbable material (Maxon; Covidien, USA).
Left nephrectomy was done and histologic examination was conducted. Additionally, involved fistula was sutured. In dissected caudal pole of left kidney, two yellow suture materials were identified. Histopathological examination showed granulomatous inflammation and fibrosis in caudal pole of left kidney but no remarkable features were identified in the other region which indicated chronic inflammation due to suture material as foreign body (Fig. 4). Microscopic examination indicated granulomatous suppurative inflammation and necrosis in the lesion. Massive macrophages and polymorphonuclear leukocytes with fibrotic tissues infiltrated into the kidney parenchyma. The type of suture material used earlier 
was unknown as there were no medical records.

Postoperatively, the patient received cefazolin $(25 \mathrm{mg} / \mathrm{kg}$, IV, 12 hourly), carprofen $(2.2 \mathrm{mg} / \mathrm{kg}, \mathrm{SC}, 12$ hourly; Rimadyl, Zoetis Inc., USA), tramadol (4 mg/kg, IV, 12 hourly; Tridol, Yuhan, Seoul, Korea), famotidine (0.5 mg/kg, IV, 12 hourly; Gaster, Donga ST, Seoul, Korea). Urethral catheter was placed for four days and measured above $1 \mathrm{~mL} / \mathrm{kg} / \mathrm{h}$ throughout hospitalization. Compete blood count and serum chemistry showed normal results for protein and globulin. However, mild anemia, neutrophilia, and monocytosis were noted. The patient recovered unremarkably and was discharged four days post-surgery.

\section{Discussion}

Retroperitoneal abscess with or without fistula associated with foreign body is widely reported $(5,22,23)$. To the best of our knowledge, there is reported case of retroperitoneal fistula induced by suture material involving the kidney. This case shows non-specific clinical signs after one year of previous OHE. Recording surgical history, ultrasonography, and computed tomographic examinations were essential for establishing diagnosis and treatment.

The etiology is mostly associated with foreign bodies, but they can pose diagnostic challenges (15). Diagnosis of the suture granuloma or abscess is demanding, especially in dogs developing the condition after a significant interval post-hysterectomy (8). The surgery and the appearance of granulomas or abscess due to foreign bodies may take several months to years (7). The clinical signs are non-specific, with an abdominal mass or draining tract in an animal with a suspicious history of previous surgery (18). This case also presented with subcutaneous abscess after one year of previous $\mathrm{OHE}$ and the lesion relapsed after antibiotics discontinuation. The hematological and serum biochemical abnormalities may involve neutrophilia with a left shift, toxic cellular changes, monocytosis, nonregenerative anemia, hyperglobulinemia or azotemia (8). However, this case was only related to hyperglobulinemia. Cytological examination showed chronic inflammation including neutrophils, plasma cells, and macrophages corresponding to the features in suture abscess as described previously (25).

The diagnostic support to retained surgical foreign bodies may include sinography or fistulography with cinefluoroscopy, ultrasonography, and computed tomography (15). Abdominal ultrasonography is a superior diagnostic aid for diagnosing retroperitoneal abcesses than radiography (22). Ultrasonography is an effective diagnostic procedure for identification of nonradiopaque foreign bodies and has been successfully used in the thorax, abdomen, eyes, and skin $(1,9,13,14,17,27)$. Identification of retained foreign bodies is often enhanced as a hypoechoic region associated with a focal inflammatory change (10) as observed in this case. Computed tomography was also helpful for diagnosis and surgical planning.

As described above, these features are similar to human medicine. Case reports about foreign body reactions to suture materials are rare (12). This could be due to short surgeons' visits or reactions to suture material being considered as non-specific surgical complications (26). Although reported in human medicine, etiology of retroperitoneal fistula occurrence is well described. Fistula formation may occur by fibrous reaction, intra- or postoperative contamination, or suture material (29). They are caused by response to non-absorbable suture material or inappropriate aseptic technique (30). The majority of fistulas present with spontaneous draining tract through the lumbar region following the lumbar triangle and the lumbar quadrilateral which are the lowest resistance points and connecting the perirenal tissues and urinary system with the external environment through a fistulous pathway $(2,21,28)$. In small animals, fistula frequently occurs in the lumbar, paralumbar, and flank areas $(6,16,19)$. Cases of fistula connection between kidney and retroperitoneal skin have been reported in dogs but without foreign body association $(20,24)$. As described previously, in humans and dogs, fistula may affect kidney through suppurative lesion which requires surgical resection of the affected kidney for complete resolution $(2,15,20,24)$.

The limitation of this case was difficulty in identifying the type of suture material used previously during OHE as there were no medical records.

\section{Conclusions}

In conclusion, eight-year-old spayed female poodle dog presented with bilateral subcutaneous masses after two years of previous ovariohysterectomy. Diagnostic procedures including history recording, cytologic examination, abdominal ultrasonography, computed tomography, and exploratory celiotomy were carried out. Diagnosis was established as retroperitoneal suppurative fistula involving left kidney induced by suture material. This case report describes diagnostic procedure and surgical treatment of fistula induced by suture material used in ovariohysterectomy after long interval.

\section{Conflicts of Interest}

The authors have no conflicting interests. 


\section{References}

1. Adams R, Nixon A, Hager D. Use of intraoperative ultrasonography to identify a cervical foreign body. A case report. Vet Surg 1987; 16: 384-388.

2. Ansari MS, Singh I, Dogra PN. Spontaneous nephrocutaneous fistula--2 unusual case reports with review of literature. Int Urol Nephrol 2004; 36: 239-243.

3. Armbrust LJ, Biller DS, Radlinsky MG, Hoskinson JJ. Ultrasonographic diagnosis of foreign bodies associated with chronic draining tracts and abscesses in dogs. Vet Radiol Ultrasound 2003; 44: 66-70.

4. Blood DC, Studdert VP. Baillière's comprehensive veterinary dictionary. London: Baillière Tindall. 1993.

5. Boza S, Lucas X, Zarelli M, Soler M, Belda E, Agut A. Late abscess formation caused by silk suture following hysterectomy in a female dog. Reprod Domest Anim 2010; 45: 934-936.

6. Brennan KE, Ihrke PJ. Grass awn migration in dogs and cats: a retrospective study of 182 cases. J Am Vet Med Assoc 1983; 182: 1201-1204.

7. Calkins CM, St Peter SD, Balcom A, Murphy PJ. Late abscess formation following indirect hernia repair utilizing silk suture. Pediatr Surg Int 2007; 23: 349-352.

8. Campbell BG. Omentalization of a nonresectable uterine stump abscess in a dog. J Am Vet Med Assoc 2004; 224: 1799-1803, 1788.

9. Cartee RE, Rumph PF. Ultrasonographic detection of fistulous tracts and foreign objects in muscles of horses. J Am Vet Med Assoc 1984; 184: 1127-1132.

10. Craychee TJ. Ultrasonographic evaluation of equine musculoskeletal injury. In: Nyland TG, Mattoon JS, editors. Veterinary diagnostic ultrasound. Philadelphia: Saunders. 1995.

11. Daigle JC, Kerwin S, Foil CS, Merchant SR. Draining tracts and nodules in dogs and cats. Clin Tech Small Anim Pract 2001; 16: 214-218.

12. Farrar MJ, Binns MS. Inflammatory reaction to subcuticular Vicryl suture following tuberculin test. Br J Plast Surg 1997; 50: 665666.

13. Farrow CS. Musculoskeletal system. In: Green RW, editor. Small animal ultrasound. Philadelphia: Lippincott-Raven. 1996.

14. Fornage BD, Nasca S, Durville A. Sonographic detection and three-dimensional localization of a metallic foreign body in soft-tissues in a dog. Br Vet J 1987; 143: 278-282.

15. Frank JD, Stanley BJ. Enterocutaneous fistula in a dog secondary to an intraperitoneal gauze foreign body. J Am Anim Hosp Assoc
2009; 45: 84-88

16. Frendin J, Funkquist B, Hansson K, Lönnemark M, Carlsten J. Diagnostic imaging of foreign body reactions in dogs with diffuse back pain. J Small Anim Pract 1999; 40: 278-285.

17. Gooding GA, Hardiman T, Sumers M, Stess R, Graf P, Grunfeld C. Sonography of the hand and foot in foreign body detection. $J$ Ultrasound Med 1987; 6: 441-447.

18. Kirby BM. Peritoneum and retroperitoneum. In: Tobias KM, editor. Veterinary surgery: small animal. St. Louis: Elsevier Saunders. 2012: 1391-1423.

19. Lamb CR, White RN, McEvoy FJ. Sinography in the investigation of draining tracts in small animals: retrospective review of 25 cases. Vet Surg 1994; 23: 129-134.

20. Lobetti RG, Irvine-Smith GS. Nephro-cutaneous fistula in a dog. S Afr Vet Assoc 2006; 77: 40-41.

21. Macchi V, Porzionato A, Morra A, Picardi EEE, Stecco C, Loukas M, et al. The triangles of Grynfeltt and Petit and the lumbar tunnel: an anatomo-radiologic study. Hernia 2017; 21: 369-376.

22. Marvel SJ, MacPhail CM. Retroperitoneal abscesses in seven dogs. J Am Anim Hosp Assoc 2013; 49: 378-384.

23. Murphy ST, Newell SM, Burrows CF. What is your diagnosis? Foreign body in the abdomen. J Am Vet Med Assoc 1998; 212: 195 196.

24. Oh KS, Son $\mathrm{CH}$, Park CH. Spontaneous nephro-cutaneous fistula with pyonephrosis in two dogs: two case reports. Vet Med 2017; 62: 44-47.

25. Park $\mathrm{CH}$, Kwon YS, Isomura H. A histopathological study on suture granuloma in neutralized dogs. Korean J Vet Res 2002; 42: 419-423.

26. Perez A, Lozano M, Gomez M, Orta M, Minguez A, Rdez Mosquera $\mathrm{M}$, et al. Hypersensitivity to suture. Allergy 1995; 50 (26 Suppl): 371.

27. Radlinsky MG, Homco LD, Blount WC. Ultrasonographic diagnosis--radiolucent pulmonary foreign body. Vet Radiol Ultrasound 1998; 39: 150-153.

28. Sarmiento Robles C, Blasco Carratalá F, Herrera Fernández F, Chica Rusillo A, Ostalé Gómez J. [Spontaneous nephrocutaneous fistula. Report of a case and review of the literature]. Arch Esp Urol 1990; 43: 411-413. Spanish.

29. Sergeant PT, Derom F. Aorto-enteric fistula as clinical entity. Acta Chir Belg 1978; 77: 187-194.

30. van Goethem B, Schaefers-Okkens A, Kirpensteijn J. Making a rational choice between ovariectomy and ovariohysterectomy in the dog: a discussion of the benefits of either technique. Vet Surg 2006; 35: 136-143. 